\title{
Real Time Acquisition and Calibration of S/TEM Probe Current Measurement Simultaneously with Any Imaging or Spectroscopic Signal
}

\author{
Stephen D. House ${ }^{1}$, C. Tom Schamp ${ }^{2,3}$, Judith C. Yang ${ }^{1}$ \\ 1. Department of Chemical and Petroleum Engineering, University of Pittsburgh, Pittsburgh, PA (USA) \\ 2. MAS, LLC, Suwanee, GA (USA) \\ 3. (Previous) Hitachi High-Technologies America Inc., Dallas, TX (USA)
}

Advances in S/TEM optics and detectors have spurred increasing interest and capabilities in quantifying imaging and spectroscopic signals from these instruments. Quantitative atomic-resolution ADF-STEM $[1,2]$, EDXS $[3,4]$, and EELS [5,6] techniques offer the potential for accurately recovering 3D structure and composition information at nano- and atomic-scale resolutions on absolute scales. Critical to these techniques is knowledge of the probe current incident upon the specimen. Increasing the accuracy and precision of this measurement correspondingly increases the accuracy and precision with which one can quantify the number and type of atoms. To date, measurement of probe current is performed at the beginning of the session or experiment and assumed to remain constant throughout. This may be acceptable for certain electron emission sources (e.g., high-quality Schottky emitters), but as the field continues to advance even greater accuracy is needed, and this assumption will be increasingly problematic. This is especially true for experiments with long acquisition times, such as for in situ $\mathrm{S} / \mathrm{TEM}$ techniques. Even now, the constant-current assumption is unsuitable for cold field-emission guns (CFEGs). CFEGs offer superior analytic performance to Schottky emitters: higher brightness, greater spatial coherence, and a significantly lower energy spread which together yield better spatial and energy resolutions. However, this comes at the cost of reduced stability of the beam current, which decays non-linearly with time and can notably vary across a single image (e.g., Figure 1b-c). For example, for the instrument on which this work was performed, the mean spread (max-min) in probe current across a single image was $\sim 6 \%$. If CFEGs are to be employed for accurate quantitative STEM and spectroscopy, and to push the field as a whole, a new approach to current measurement is needed.

The constant-current assumption's continued usage is because existing methods for measuring probe current are incompatible with the simultaneous acquisition of other signals. The most accurate method for measuring the probe current is to use a Faraday cup, but this requires collection of the whole probe, preventing the beam from also being used for imaging or EELS. Further, if a dedicated Faraday cup holder is used, the entire specimen holder is replaced. Other methods for measuring probe current in S/TEM include calibrating the output of the CCD, STEM detector, phosphor screen, or GIF drift tube while the probe is incident upon it [1,7]. These, however, all still require the entirety of an unscattered beam to be collected and so cannot be employed while a specimen is inserted in the path of the beam.

Here we present a technique for measuring the probe current in real time concurrently with any imaging or spectroscopic signal acquisition desired. The feasibility of the approach has been demonstrated using a Hitachi HD-2700A STEM. A systematic study of the impact and origin of errors in each step of the methodology was performed and will be discussed along with directions for continued improvement. In this approach, sub-picoamp-sensitivity signal collection hardware is attached to the probe-forming aperture of the microscope. Simultaneous acquisition of this aperture current with the probe current (measured using a Faraday cup TEM holder) confirmed a reliable and repeatable linear relationship between the aperture and probe currents (e.g., Figure 1d), despite any non-linear excursions of the probe 
current itself. Having validated the use of the aperture signal for measuring probe current, the aperture current was converted into a voltage in the appropriate range required for optimal linear read-in by the analog-to-digital (ADC) convertor used by Gatan's Digiscan II hardware. This enables the aperture current - and thus the probe current - to be collected in time-synced real time on a pixel-by-pixel basis with any signal being collected by the (ubiquitous) Gatan Microscopy Suite (Digital Micrograph) software (Figure 1e). The resulting "probe current image" is acquired and readable in the same manner as any other image acquired in GMS. The scripting potential of Digital Micrograph introduces the potential for users to do image processing or calibrations with this signal on-the-fly. [8]

\section{References:}

[1] JM LeBeau and S Stemmer, Ultramicroscopy 108 (2008), pp. 1653-1658.

[2] L Jones, IOP Conference Series: Materials Science and Engineering 109 (2016), p. 012008.

[3] M Watanabe and DB Williams, Journal of Microscopy 221 (2006), pp. 89-109.

[4] Z Chen, et al., Ultramicroscopy 157 (2015), pp. 21-26.

[5] R Leapman, "EELS Quantitative Analysis, in: Transmission Electron Energy Loss Spectrometry in Materials Science and The EELS Atlas", ed. CC Ahn, (Wiley-VCH Verlag, Weinheim), pp. 49-96.

[6] HL Xin, et al., Ultramicroscopy 139 (2014), pp. 38-46.

[7] DRG Mitchell and MJB Nancarrow, Microscopy Research and Technique 78 (2015), pp. 886-893.

[8] This work was supported by NSF DMREF through contract No CHE-1534630, Hitachi High-

Technologies Corp., and the ETEM Catalysis Consortium (ECC, funded through U.Pitt). The authors would like to acknowledge the usage of the STEM at the Georgia Institute of Technology, as well as R.D. Twesten (Gatan) for information about the Digiscan hardware.

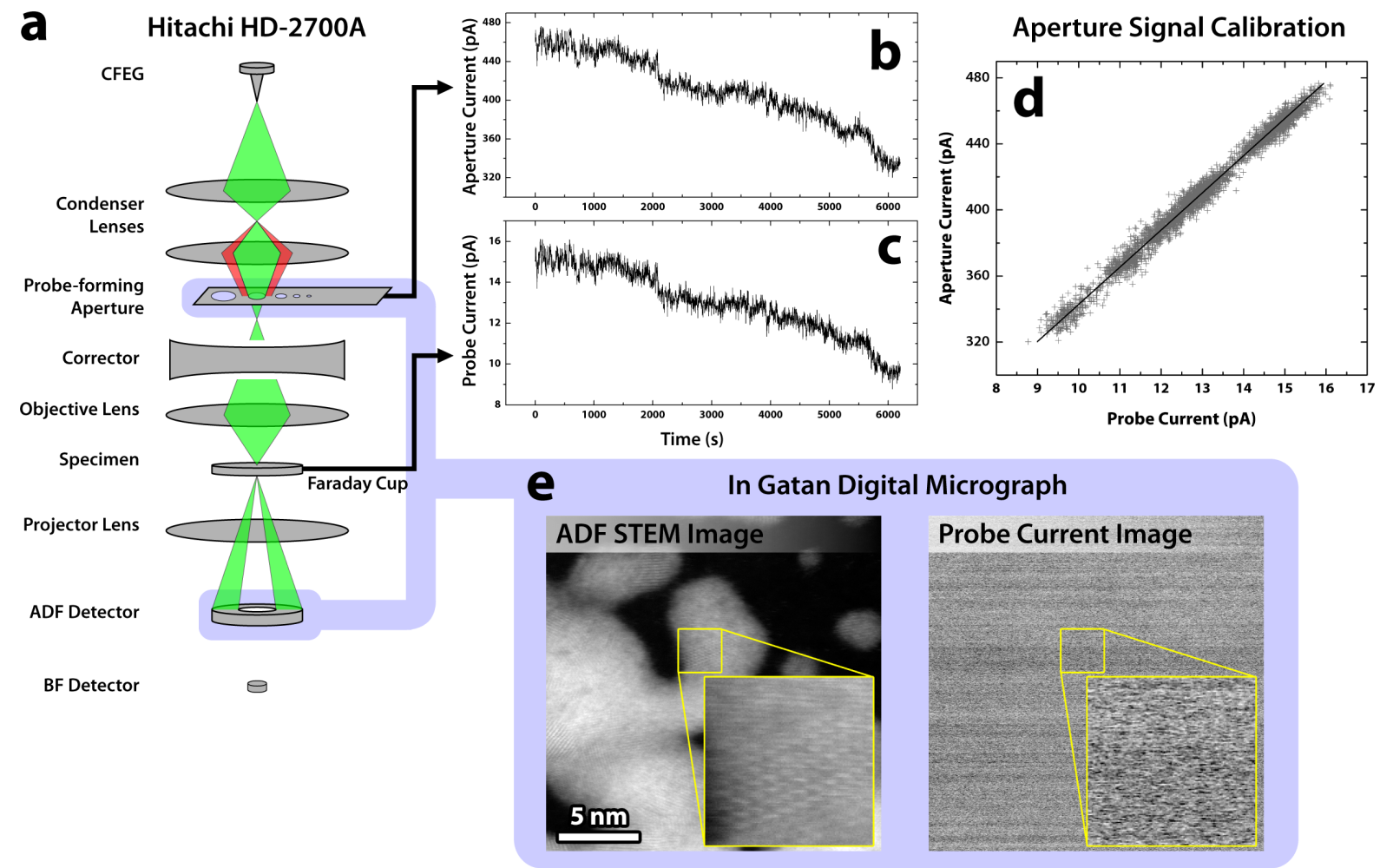

Figure 1. (a) Simplified diagram of the relevant internals of the HD-2700A STEM. (b-c) Example of concurrently measured probe and condenser aperture currents revealing (d) the linear relationship between the two. A Faraday cup holder was used to make these measurements. (e) Simultaneously acquired ADF-STEM and aperture current images. 